\title{
Medidas diretas de atividade física em crianças e cumprimento das recomendações da Organização Mundial de Saúde
}

http://dx.doi.org/10.11606/1807-5509201900010049

\author{
Alessandro H. Nicolai RÉ* \\ Anderson Henry FEITOZA** \\ Carlos Bandeira de MELLO MONTEIRO* \\ Elisangela da SILVA* \\ Mariana C. TUDELA* \\ Maria Teresa CATTUZZO** \\ ${ }^{*}$ Escola de \\ Artes, Ciências \\ e Humanidades, \\ Universidade de São \\ Paulo, São Paulo, SP \\ Brasil. \\ **Universidade de \\ Pernambuco, Recife \\ PE, Brasil.
}

\section{Resumo}

Os objetivos deste estudo foram verificar a quantidade e intensidade de prática de atividade física (AF) em crianças de baixo nível socioeconômico, e avaliar o cumprimento das recomendações da Organização Mundial de Saúde (AF moderada a vigorosa $\geq 60$ minutos diários) nos dias de semana e finais de semana. Participaram do estudo 174 crianças (49\% meninas) entre 7 e 10 anos de idade, residentes em área de vulnerabilidade social. 0 tempo e intensidade da AF foram medidos com acelerômetros ( $w G T 3 X+$ ), durante 7 dias consecutivos, e para caracterizar a AF moderada a vigorosa (AFMV) foi utilizado um ponto de corte $\geq 2296$ contagens por minuto. As comparações entre os sexos foram realizadas por meio da Análise de Variância, utilizando-se ainda o Teste t para medidas dependentes para as comparações entre a AFMV praticada durante a semana e os finais de semana, e o teste qui-quadrado para verificar as associações entre as variáveis sexo e cumprimento das diretrizes internacionais de atividade física. Em média, os meninos realizaram mais AFMV do que as meninas $(p<0,01)$, tanto em dias de semana $(77 \times 58$ minutos por dia) como nos finais de semana ( $71 \times 50$ minutos por dia). Durante os dias de semana, 59\% das crianças atingiram as recomendações ( $72 \%$ dos meninos e $42 \%$ das meninas; $p<0,001$ ) e apenas $47 \%$ ( $57 \%$ dos meninos e $34 \%$ das meninas; $p<0,001$ ) atingiram as recomendações durante os finais de semana. Esses dados reforçam a necessidade de ampliação da oferta de atividades físicas voltadas à realidade sociocultural da criança, com atenção especial para as meninas e para um maior envolvimento das famílias, particularmente durante os finais de semana.

Palavras-Chave: Promoção da saúde; Acelerometria; Atividade física; Crianças.

\section{Introdução}

A prática regular de atividade física $(\mathrm{AF})$ durante a infância e a adolescência favorece o desenvolvimento motor, cognitivo e afetivo-social, além de ser um forte preditor de continuidade da prática na idade adulta, com consequências positivas para a qualidade de vida e a prevenção de doenças crônico-degenerativas associadas à obesidade, diabetes, alguns tipos de câncer e problemas cardio-vascular-respiratórios ${ }^{1-3}$. Diretrizes atuais de saúde sugerem que, para um estilo de vida saudável, crianças maiores de cinco anos de idade devem acumular, no mínimo, 60 minutos diários de atividade física em intensidade moderada a vigorosa (AFMV) ${ }^{2,4}$.

De modo geral, pesquisas recentes têm reportado valores inferiores de prática de $\mathrm{AF}$ em crianças e adolescentes de baixo nível socioeconômico5 Todavia, em amplo estudo de revisão, Stalsberg e Pedersen ${ }^{6}$ identificaram forte divergência entre as pesquisas, com $42 \%$ dos estudos incluídos reportando ausência de associação ou até mesmo uma associação negativa entre a prática de $\mathrm{AF}$ e o nível socioeconômico. $\mathrm{Na}$ realidade, parece que a vulnerabilidade social, não necessariamente dependente do nível socioeconômico, é um fator que pode restringir a prática de AF devido aos prejuízos associados a fatores como a violência e a falta de espaços públicos apropriados ${ }^{7-9}$ que, entre outras consequências, podem contribuir para a diminuição das experiências motoras e gerar também 
atrasos no desenvolvimento motor. Corroborando essa tendência, em pesquisa recente realizada com crianças em situação de vulnerabilidade social na cidade de São Paulo, foi detectado um atraso crescente nos indicadores de desenvolvimento motor dos 3 aos 10 anos de idade, sugerindo um efeito cumulativo das restriçóes ambientais na aquisição de $\mathrm{HMF}^{10}$. Outros estudos com crianças brasileiras têm encontrado resultados semelhantes ${ }^{11,12}$.

Assim, são necessárias pesquisas adicionais que considerem o contexto em que as crianças de baixo nível socioeconômico estão inseridas ${ }^{13-15}$, uma vez que a pobreza, especialmente em grandes centros urbanos, é um fenômeno que apresenta forte componente territorial, caracterizado por processos de segregaçáo socioespacial ${ }^{16-18}$ que devem ser levados em conta no entendimento da prática de $\mathrm{AF}^{6-8}$. Concomitantemente, o conhecimento dos valores de prática de $\mathrm{AF}$

\section{Método}

\section{Participantes}

Os dados do presente estudo pertencem a uma amostra aleatória de 1.370 escolares entre 3 e 14 anos de idade, participantes do estudo longitudinal "Associações entre prática de atividade física, aptidão física e coordenação motora em crianças e adolescentes". Para a presente pesquisa, foram incluídas 174 crianças aparentemente saudáveis entre 7 e 10 anos de idade $(85$ meninas e 89 meninos; média \pm DP: idade $8,2 \pm 0,8$ anos; peso $29,2 \pm 7,7 \mathrm{~kg}$; estatura $130,1 \pm 7,5 \mathrm{~cm}$ ) que estudam em escola pública e participam de aulas de educação física duas vezes por semana, conforme currículo escolar. O estudo foi aprovado pelo comitê de Ética da EACH/USP (CAAE: 24766913.0.0000.5390). Os pais ou responsáveis assinaram um formulário de consentimento livre e esclarecido e as crianças forneceram assentimento para participar da pesquisa.

\section{Instrumentos e procedimentos}

O tempo e a intensidade da prática de $\mathrm{AF}$ foram mensurados de modo direto, com a utilizaçáo de acelerômetros (ActiGraph GT3X+, Pensacola, FL, USA) por 7 dias consecutivos. Os pais/responsáveis receberam uma planilha com informaçóes detalhadas sobre o funcionamento do aparelho e foram nessa população é importante para a geração de dados que fundamentem a elaboração de projetos de incentivo à prática de $\mathrm{AF}^{19,20} \mathrm{e}$, potencialmente, contribuam com a reduçấo das taxas de sedentarismo e a consequente prevenção de doenças crônico-degenerativas, possibilitando ainda uma maior integração social e elevação da qualidade de vida.

Portanto, os objetivos deste estudo foram: (a) verificar a quantidade e intensidade de prática de AF em crianças de ambos os sexos em situaçáo de vulnerabilidade social e (b) avaliar o cumprimento das recomendaçóes internacionais de prática de AF (AFMV $\geq 60$ minutos diários) nos dias de semana e finais de semana. Como hipótese, em função das limitaçóes ambientais associadas ao baixo nível socioeconômico em grandes centros urbanos, é esperado encontrar baixos valores de prática de $\mathrm{AF}$ nessas crianças.

instruídos da necessidade de manutenção dos hábitos normais das crianças. $\mathrm{O}$ monitor foi colocado na altura do quadril, preso e protegido por um cinto, e foi retirado apenas para tomar banho e dormir. Ligaçôes telefônicas e mensagens de texto via telefone celular foram utilizadas para responder dúvidas dos pais sobre do uso dos acelerômetros.

As atividades foram registradas no aparelho em intervalos (epoch) de 15 segundos e cada criança foi monitorada durante sete dias consecutivos (terçafeira a segunda-feira). O tempo de não utilização do aparelho (non-wear time) foi definido como 60 minutos consecutivos de contagem igual a zero, permitindo-se 2 minutos de interrupçáo com valores acima de zero. Foram incluídas no estudo as crianças que usaram o acelerômetro, no mínimo, 500 minutos por dia durante 3 dias (incluindo pelo menos 1 dia do final de semana), de acordo com critérios sugeridos em pesquisas recentes ${ }^{21-23}$.

Os dados foram interpretados utilizando pontos de corte propostos por EvENSON et al. ${ }^{24}$ : 0-100 contagens por minuto (cpm) de tempo para sedentarismo; 101-2295 para AF leve; 2296-4011 para AF moderada e a partir de 4012 para AF vigorosa. A partir desses pontos de corte, foi calculado o tempo médio de prática de $\mathrm{AF}$ em diferentes intensidades nos dias válidos de uso do acelerômetro e, tendo como base as diretrizes mundiais de prática de $\mathrm{AF}^{2,4}$, foi calculada a proporção de crianças 
que tiveram uma média diária de $\mathrm{AFMV} \geq 60$ minutos. Adicionalmente, utilizando uma interpretação mais restritiva das diretrizes ${ }^{21}$, foi calculada a porcentagem de escolares que praticaram $\geq 60$ minutos de AFMV em todos os dias válidos de uso do acelerômetro e a porcentagem de dias válidos de uso em que as diretrizes foram atingidas.

Como indicador do nível socioeconômico, primeiramente foi verificado o local de habitaçáo dos participantes dessa pesquisa por meio do Código de Endereçamento Postal (CEP) das residências e escolas. A partir dessa informaçáo, foi utilizado o mapa de vulnerabilidade social da cidade de São Paulo ${ }^{16,25,26}$, que adota um critério territorial e considera as condiçóes do local de habitação associados ao acesso a serviços públicos, indicadores de violência urbana, privação econômica das famílias e acesso à informação e cultura. O CEP na cidade de São Paulo informa, com exatidão, o local de habitaçáa ${ }^{27}$, e desse modo foi identificado que todos os participantes da presente pesquisa estudam e residem em favelas no bairro de Ermelino Matarazzo, locais considerados de elevada vulnerabilidade social e baixo nível socioeconômico ${ }^{16,25,26}$.

Para a caracterização dos participantes, além das informaçóes demográficas sobre o sexo, data de nascimento e local de habitação, também foram mensuradas a massa corporal (precisão de $100 \mathrm{~g}$ ) e a estatura (precisão de $0,1 \mathrm{~cm}$ ) utilizando-se uma balança digital com estadiômetro acoplado (Balmak Premium, SP, Brasil), e calculado o índice de massa corporal (massa corporal, $\mathrm{kg} x$ estatura, $\mathrm{m}^{-2}$ ), seguindo rigorosamente o protocolo descrito por LoHMAN et al. ${ }^{28}$.

\section{Análise de dados}

Para a análise de dados, os níveis de AF de cada criança foram calculados a partir do tempo médio despendido nas intensidades leve, moderada e vigorosa nos dias válidos de uso do acelerômetro. Para examinar a distribuiçáo dos padróes de AF, os dados foram calculados considerando apenas os dias de semana (segunda a sexta-feira), os dias de final de semana (sábado e domingo) e a semana completa. $\mathrm{Na}$ análise exploratória dos dados, foi confirmada a existência de distribuição normal e de igualdade de variâncias, por meio dos testes de Kolmogorov-Smirnov e de Levene, respectivamente, de acordo com as recomendaçóes da literatura ${ }^{29}$. Ainda na fase exploratória, a análise de variância (ANOVA) foi utilizada para comparar o tempo despendido em AFMV em cada valor discreto de idade ( 7 anos, 8 anos, 9 anos e 10 anos) e também entre a série da criança no ensino fundamental ( $2^{\circ}$ ano, $3^{\circ}$ ano e $4^{\circ}$ ano). Uma vez que não houve diferença estatisticamente significante entre esses grupos no tempo despendido em AFMV, as análises subsequentes descritas na presente pesquisa foram conduzidas com as idades e séries escolares unificadas, considerando-se apenas os agrupamentos de acordo com o sexo.

Dessa forma, foram computadas medidas descritivas [média e desvio-padrão (DP)] acerca de prática de $\mathrm{AF}$ de meninos e meninas, com as comparações entre os sexos sendo realizadas por meio da ANOVA com um fator (sexo). Também foi utilizado o Teste $t$ para medidas dependentes para verificar as eventuais diferenças entre o tempo despendido em AFMV durante a semana e aos finais de semana e o teste qui-quadrado foi utilizado para verificar as associaçōes entre as variáveis sexo e cumprimento das diretrizes internacionais de AF. As análises estatísticas foram realizadas com a utilização do software SPSS versão 22 e o nível de significância estabelecido foi $5 \%$.

\section{Resultados}

Entre as 174 crianças que usaram o acelerômetro, 141 (62 meninas, idade $=8,4 \pm 0,97$ anos e 79 meninos, idade $=8,3 \pm 0,94$ anos) atingiram o critério de tempo mínimo de uso e foram incluídas no estudo. O tempo médio de uso do acelerômetro foi de 4,6 dias durante a semana com uso de $786 \mathrm{~min} /$ dia e 1,8 dias durante o final de semana, com uso de $714 \mathrm{~min} /$ dia. Não houve diferença estatisticamente significante entre os sexos nos valores de índice de massa corporal (IMC) e idade. Um possível viés decorrente da seleção de escolares em função dos critérios de tempo mínimo de uso do acelerômetro foi verificado comparando-se o IMC e a idade entre os grupos incluídos e não incluídos no estudo; não houve diferença entre os grupos (dados não descritos).

$\mathrm{Na}$ TABELA 1 são apresentados os valores médios de prática de AF nos dias de semana, finais de semana e na semana completa, de acordo com o sexo. Os meninos despenderam mais tempo em AFMV do que as meninas tanto nos dias de semana 
a: diferença entre os sexos (ANOVA, $\mathrm{p}<0,003)$;

$b$ : diferença entre dias de semana e final de semana (Teste t para medidas dependentes, $p<0,04)$. (meninos 77,1 min $\mathrm{x}$ meninas 58,1 min; $\mathrm{p}<0,01$ ) como nos dias de final de semana (meninos 70,8 min $\mathrm{x}$ meninas 49,8 min; $\mathrm{p}<0,01$ ). Além disso, a média do tempo gasto em AFMV das meninas está abaixo das recomendaçóes internacionais. Em ambos os sexos, o tempo gasto em AFMV foi significantemente superior nos dias de semana em comparação com os dias de final de semana.

Na FIGURA 1, é apresentada a proporção de crianças que atingem as recomendaçóes internacionais de prática de $\mathrm{AF}$ ( $\geq 60$ min diários de AFMV), considerando a média de AFMV computada nos dias válidos de uso do acelerômetro. Analisando a semana completa, $54,6 \%$ das crianças atingem as recomendaçóes, sendo a quantidade de meninos $(68,4 \%)$ significantemente superior à de meninas $(37,1 \%)\left(c^{2}=13,69, \mathrm{p}<0,001\right)$. Durante os dias de semana, $58,9 \%$ das crianças atingem as recomendaçóes $(72,2 \%$ dos meninos e $41,9 \%$ das meninas; $\left.\mathrm{c}^{2}=13,09, \mathrm{p}<0,001\right)$ e apenas $46,8 \%$ das crianças $(57,0 \%$ dos meninos e $33,9 \%$ das meninas; $\left.c^{2}=7,44, p<0,01\right)$ atingem as recomendaçōes durante os dias de final de semana.

Utilizando uma interpretação mais rigorosa das diretrizes internacionais, $14 \%$ das crianças praticaram $\geq 60$ min de AFMV em todos os dias válidos de uso do acelerômetro (23\% dos meninos e apenas 3\% das meninas, $\mathrm{p}<0,001)$. A prática de AFMV foi $\geq 60$ minutos em $51 \%$ dos dias válidos de uso $(61 \%$ dos dias válidos dos meninos e $38 \%$ das meninas, $\mathrm{p}<0,001)$.

TABELA 1 - Média e desvio-padrão (DP) do tempo gasto em AFMV nos dias de semana, finais de semana e semana completa

\begin{tabular}{|c|c|c|c|c|c|c|c|}
\hline \multirow{2}{*}{ Medida } & \multicolumn{3}{|c|}{ Meninas $(n=62)$} & \multicolumn{3}{|c|}{ Meninos $(\mathbf{n}=79)$} & \multirow{2}{*}{$\begin{array}{c}\text { Total } \\
(\mathrm{n}=141)\end{array}$} \\
\hline & Média (DP) & Mín. & Máx. & Média (DP) & Mín. & Máx. & \\
\hline \multicolumn{8}{|l|}{ AF leve (minutos/dia) } \\
\hline Dias de semana & $297,4(44,3)^{b}$ & 53,1 & 231,2 & $299,7(51,4)^{\mathrm{b}}$ & 55,7 & 237,1 & $298,7(48,2)^{b}$ \\
\hline Final de semana & $273,0(58,9)^{\mathrm{b}}$ & 50,3 & 260,1 & $278,9(64,8)^{\mathrm{b}}$ & 52,2 & 279,7 & $276,3(62,2)^{b}$ \\
\hline Semana completa & $291,2(42,1)$ & 49,0 & 266,2 & $294,2(49,1)$ & 54,8 & 280,3 & $292,9(46,0)$ \\
\hline \multicolumn{8}{|c|}{ AF moderada (minutos/dia) } \\
\hline Dias de semana & $43,1(12,6)^{\mathrm{a}, \mathrm{b}}$ & 10,9 & 77,3 & $55,1(16,0)^{\mathrm{a}, \mathrm{b}}$ & 9,9 & 84,6 & $49,8(15,7)^{b}$ \\
\hline Final de semana & $36,4(14,8)^{a, b}$ & 9,0 & 62,2 & $50,7(20,7)^{\mathrm{a}, \mathrm{b}}$ & 14,1 & 105,2 & $44,4(19,6)^{b}$ \\
\hline Semana completa & $41,4(11,6)^{\mathrm{a}}$ & 15,3 & 55,2 & $53,8(15,8)^{\mathrm{a}}$ & 11,2 & 95,3 & $48,4(15,4)$ \\
\hline \multicolumn{8}{|c|}{ AF vigorosa (minutos/dia) } \\
\hline Dias de semana & $15,1(7,2)^{a}$ & 11,9 & 46,2 & $22,0(11,7)^{a}$ & 13,3 & 73,4 & $18,9(10,5)^{b}$ \\
\hline Final de semana & $13,4(9,9)^{a}$ & 8,1 & 47,1 & $20,1(13,9)^{a}$ & 14,6 & 86,2 & $17,2(12,7)^{\mathrm{b}}$ \\
\hline Semana completa & $14,7(6,9)^{\mathrm{a}}$ & 9,6 & 50,3 & $21,4(11,4)^{\mathrm{a}}$ & 10,1 & 84,7 & $18,5(10,2)$ \\
\hline \multicolumn{8}{|l|}{$A F M V$ (minutos/dia) } \\
\hline Dias de semana & $58,1(18,0)^{a, b}$ & 28,3 & 113,5 & $77,1(25,6)^{\mathrm{a}, \mathrm{b}}$ & 29,9 & 129,8 & $68,8(24,4)^{\mathrm{b}}$ \\
\hline Final de semana & $49,8(22,7)^{a, b}$ & 18,2 & 109,3 & $70,8(32,9)^{\mathrm{a}, \mathrm{b}}$ & 20,0 & 150,9 & $61,5(30,6)^{b}$ \\
\hline Semana completa & $56,1(16,8)^{a}$ & 20,1 & 105,5 & $75,3(25,5)^{a}$ & 28,5 & 125,2 & $66,8(24,0)$ \\
\hline
\end{tabular}




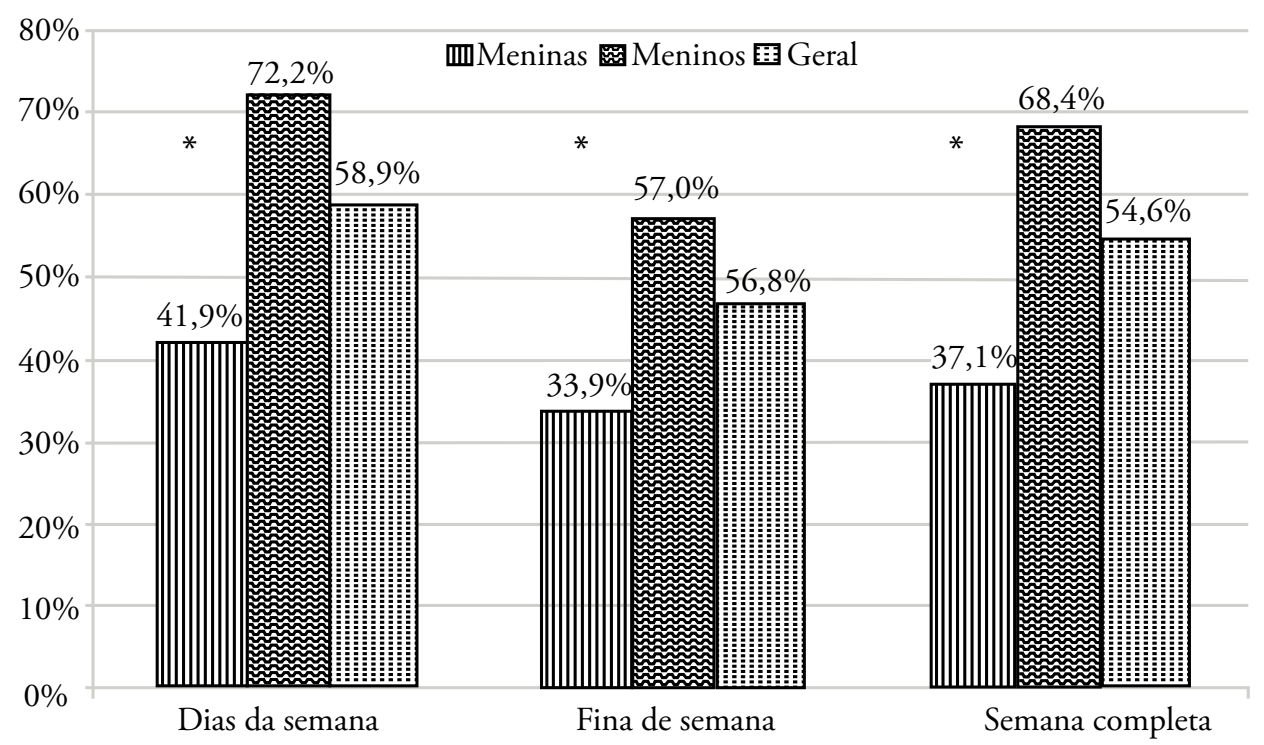

FIGURA 1 - Proporção de meninos e meninas entre 7 a 10 anos de idade que atingem as recomendações de $*: p<0,01$. prática de AFMV nos dias de semana, final de semana e semana completa.

\section{Discussão}

O objetivo principal desta pesquisa foi verificar a quantidade e intensidade de tempo dedicada à prática de AF de crianças de baixo nível socioeconômico durante os dias de semana e finais de semana e avaliar o cumprimento das recomendaçóes da Organização Mundial de Saúde ${ }^{2,4}$. A hipótese de que essas crianças tendem a apresentar baixos valores de prática de AFMV foi confirmada, principalmente nas meninas e aos finais de semana. Porém, os valores encontrados não divergem daqueles relatados em outras pesquisas com diversas populaçóes ${ }^{30-35}$. Em média, os meninos atingiram os valores recomendados de prática de AFMV e tiveram resultados superiores aos das meninas durante a semana e aos finais de semana.

Os resultados obtidos na presente pesquisa seguiram a tendência observada em outros estudos que mediram a prática de AF de modo direto e relataram superioridade de meninos em comparaçáo às meninas ${ }^{21,32-38}$ e maiores índices de prática de atividade física durante os dias de semana em ambos os sexos ${ }^{34,39,40}$. Na realidade, a proporção de crianças atingindo as diretrizes atuais de prática de AF têm grande variação em função, principalmente, dos pontos de corte utilizados para determinar as intensidades de $\mathrm{AF}^{41,42} \mathrm{e}$, portanto, na comparação entre estudos é fundamental levar em consideração este fator.

Em amplo estudo de revisão focado em publicaçôes com crianças e adolescentes europeus, Guinhouya et al. ${ }^{37}$ relataram que a proporção de crianças ( $\leq 12$ anos de idade) que atingiu as recomendaçóes de AFMV variaram entre 1\% e 100\%, dependendo principalmente do ponto de corte utilizado para definir a AFMV. Aproximadamente $78-100 \%$ das crianças atingiram as diretrizes em estudos que utilizaram um ponto de corte aproximado $>1000-1500 \mathrm{cpm}$. Em contrapartida, somente $3 \%$ a $9 \%$ das crianças europeias atingiram as diretrizes quando o ponto de corte para a AFMV foi maior que $3000 \mathrm{cpm}$ e apenas $1 \%$ das crianças foram consideradas suficientemente ativas quando o ponto de corte foi superior a $4000 \mathrm{cpm}$. Quando utilizado um ponto de corte de aproximadamente $2000 \mathrm{cpm}$ (semelhante ao presente estudo), cerca de 36-87\% das crianças europeias atingiram as recomendaçôes de AF. A TABELA 2 apresenta dados para a comparação entre estudos realizados com diversas populaçóes de faixa etária similar ao presente estudo. 
Ré AHN, et al.
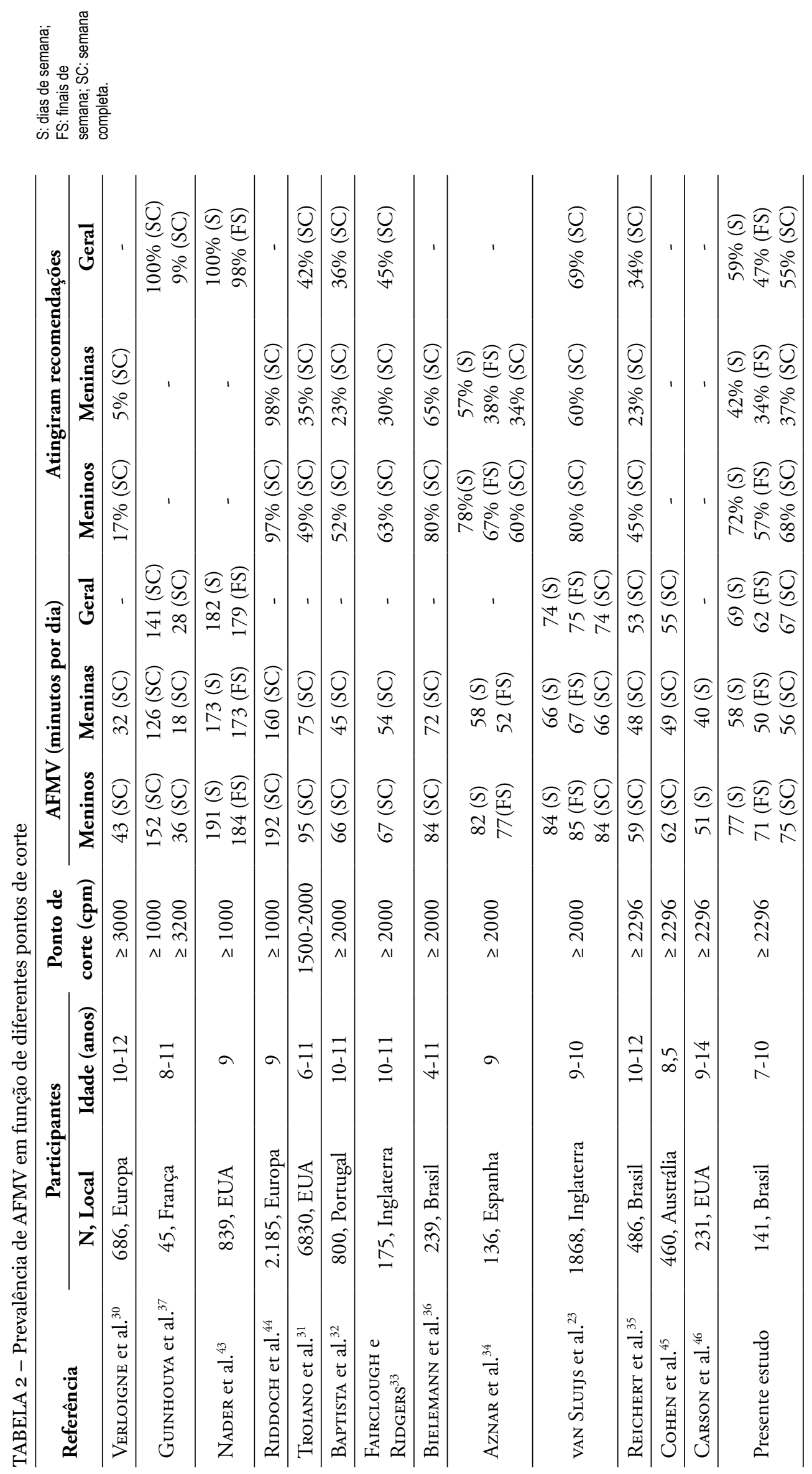
Parece que a utilização de pontos de corte acima de 1.000 ou $3.000 \mathrm{cpm}$ para caracterizar a AFMV hiperestimam ou subestimam, respectivamente, a quantidade de AFMV praticada em crianças $^{37,41}$. A proporção de crianças que atingem as diretrizes internacionais de prática de $\mathrm{AF}$ no presente estudo é compatível com os valores encontrados em estudos com diferentes populaçóes que utilizaram pontos de corte semelhantes $(\geq 2000 \mathrm{cpm})$ (TABELA 2). Reforçando essa afirmação, Cooper et al. ${ }^{21}$, em pesquisa que reuniu 27.637 participantes entre 3 e 18 anos de idade provenientes de dez países diferentes ( $82 \%$ dos participantes entre 9 e 15 anos de idade), utilizando os mesmos critérios de tempo de uso, intervalos de registro (epoch) e pontos de corte do presente estudo, relataram que apenas $9 \%$ dos meninos e $2 \%$ das meninas atingiram as diretrizes de prática de $\mathrm{AF}$ ( $\geq 60 \mathrm{~min}$ por dia) em todos os dias válidos de uso do acelerômetro; no presente estudo, $23 \%$ dos meninos e apenas 3\% das meninas atingiram as recomendações em todos os dias válidos de uso. Além disso, Cooper et al. ${ }^{21}$ relataram que os meninos atingiram as recomendaçóes em $46 \%$ dos dias válidos de uso do acelerômetro e as meninas em $22 \%$ dos dias válidos. No presente estudo, os meninos atingiram as recomendaçôes em $61 \%$ dos dias válidos de uso e as meninas em $38 \%$ dos dias válidos.

Apesar de o presente estudo ter utilizado medidas diretas de AF e pontos de corte ( $\geq 2296 \mathrm{cpm}$ ) validados e recomendados para uso em crianças e adolescentes ${ }^{21,47,48}$, ainda não existe consenso sobre as decisóes mais adequadas à análise de dados. Consequentemente, variaçóes nos protocolos de acelerometria (por exemplo, pontos de corte, tempo de uso, epochs) dificultam a comparação entre estudos e alteram os resultados obtidos $^{42,49}$. Também é necessário destacar que os acelerômetros não são usados em atividades realizadas em ambiente aquático (p. ex., natação) e subestimam as intensidades de atividades com sobrecarga (p. ex., "musculação"), ciclismo e movimentos de membros superiores. Assim, é possível que a mensuração da AF seja subestimada com o uso de acelerômetros.

Adicionalmente, entre as crianças da presente pesquisa, é provável que as oportunidades de prática de atividade física e/ou esportiva estruturada sejam escassas. Pesquisas atuais ${ }^{36,50,51}$ têm destacado que indivíduos de baixo nível socioeconômico que vivem em locais de maior vulnerabilidade social, têm menor acesso à prática de $\mathrm{AF}$ em situaçóes estruturadas e/ ou de lazer e muitas vezes as atividades físicas são realizadas em momentos de transporte ativo (p. ex., caminhar para a escola). Além disso, em geral, os meninos possuem mais apoio social e familiar para a prática de esporte e AF quando comparados com as meninas ${ }^{52,53}$; nas escolas, é comum as quadras serem ocupadas pelos meninos durante os intervalos de aulas ou até mesmo durante as aulas de Educação Física $^{54}$ e existem evidências de que meninas de baixo nível socioeconômico se envolvem menos com a prática de $\mathrm{AF}$ devido a atribuiçóes domésticas como limpar a casa, cozinhar ou cuidar dos irmáos ${ }^{52}$. Outro fator relevante que pode ter contribuído para os resultados apresentados estarem abaixo das recomendaçóes, principalmente nos finais de semana, são problemas de falta de segurança pública e escassez de espaços adequados para a prática de $\mathrm{AF}^{38,55,56}$. Provavelmente, a vulnerabilidade social impóe maiores restriçóes à prática de $\mathrm{AF}$ das meninas ${ }^{9,56,57}$, fato que pode ter contribuído para as diferenças entres sexos apresentadas no presente estudo. Os dados da presente pesquisa alertam que é desejável um aumento no tempo despendido em AF pelas crianças, particularmente em meninas de baixo nível socioeconômico expostas à vulnerabilidade social.

Hipoteticamente, fatores específicos de determinada região/país fazem com que o impacto do nível socioeconômico na prática de $\mathrm{AF}$ seja diferenciado ${ }^{58}$. Corroborando essa hipótese, pesquisas realizadas na Escócia ${ }^{59}$, Estados Unidos ${ }^{60}$ e Bélgica ${ }^{61}$ não identificaram associação entre o nível socioeconômico e a prática de $\mathrm{AF}$, indicando que o impacto do nível socioeconômico na prática de $\mathrm{AF}$ pode ser atenuado em países/locais onde o índice de vulnerabilidade social é reduzido ${ }^{55,62,63}$. Crianças de baixo nível socioeconômico expostas à vulnerabilidade social têm poucas oportunidades de prática de $\mathrm{AF}^{64,65}$ e consequentemente tendem a apresentar níveis mais baixos de AFMV em comparação com crianças não expostas à vulnerabilidade social ${ }^{8,66,67}$. Diante desse contexto, é necessário enfatizar a importância de políticas públicas de promoção da AF que atendam às necessidades específicas de determinada população ${ }^{15,60,65}$, particularmente aquelas em situação de vulnerabilidade social. Estratégias relativamente simples como a valorização da Educaçáo Física na escola e ampliação das oportunidades de prática de AF e esporte por meio da criação/utilização de espaços públicos e orientação profissional adequada, tendem a gerar resultados positivos ${ }^{14,68,69}$.

Algumas limitaçóes do presente estudo devem ser mencionadas. Considerando a relativa homogeneidade da amostra em relaçáo ao estrato socioeconômico e a regiáo de moradia, a generalização dos resultados para outras populaçóes 
não é adequada (p. ex., crianças de baixo nível socioeconômico moradoras de cidades pequenas). Outras limitaçóes importantes foram o uso de dados transversais e a falta de controle acerca das oportunidades e do tipo/qualidade de prática de $\mathrm{AF}$, sendo necessárias pesquisas adicionais para verificar esses fatores, assim como os efeitos longitudinais dos resultados observados. Não obstante, entre os principais aspectos positivos desse estudo destacamse o uso de medidas diretas para mensurar a prática de atividade física e a avaliação do cumprimento de recomendaçôes internacionais de prática de AF em dias de semana e finais de semana, sendo essa a primeira pesquisa a reportar medidas diretas de AF em crianças moradoras de uma comunidade carente da cidade de São Paulo, uma das maiores megalópoles do mundo.
Em síntese, é preocupante a quantidade de crianças que ficou abaixo dos critérios mínimos recomendados para AFMV; entre as meninas, menos da metade atingiu as recomendaçóes. Esses dados reforçam a necessidade desenvolver açóes que estimulem e ofereçam suporte à prática de AF e esporte com políticas públicas voltadas à realidade sociocultural e atenção especial para as meninas e para um maior envolvimento das famílias, particularmente durante os finais de semana. Nesse sentido, as escolas, parques, ruas de lazer, clube escolas, dentre outros espaços adequados para a prática de AF/esporte podem assumir um papel importante em possibilitar a integraçáo familiar e aquisiçáo de conhecimentos, atitudes e experiências positivas, aumentando as chances de os jovens tornarem-se adultos ativos e saudáveis.

\section{Abstract \\ Objectively measured physical activity in children and compliance with World Health Organization guidelines}

The objectives of this study were to verify the amount and intensity of physical activity (PA) in socioeconomically disadvantaged children, and to assess compliance with guidelines of the World Health Organization (PA moderate to vigorous $\geq 60$ minutes daily) in weekdays and weekends. This study included 174 children (49\% girls) aged between 7 and 10 years who lived in areas of social vulnerability. Time and intensity of PA were measured with accelerometers (wGT3X+) for 7 consecutive days. To characterize moderate to vigorous PA (MVPA) a cut point of $\geq 2296$ counts per minute was used. Comparisons between genders were performed using analysis of variance, and paired samples t-test was used for comparisons between weekdays and weekends; chi-square test was used to check the associations between the variables gender and compliance of PA guidelines. On average, boys performed more MVPA than girls ( $p<0.01)$, both on weekdays ( $77 \times 58$ minutes) and on weekends ( $71 \times 50$ minutes). During the weekdays, 59\% of children reached the guidelines ( $72 \%$ of boys and $42 \%$ of girls; $p<0.001$ ) and only $47 \%$ (57\% of boys and $34 \%$ of girls; $p<0.001$ ) met the guidelines during the weekends. These data reinforce the need to enhance the opportunities for PA, focused on the sociocultural reality of the children, with special attention to the girls and to a higher involvement of the families, particularly during weekends.

KEYWORDS: Health-promotion; Accelerometry; Physical activity; Children.

\section{Referências}

1. Kohl HW, Craig CL, Lambert EV, et al. The pandemic of physical inactivity: global action for public health. Lancet. 2012;380(9838):294-305.

2. World Health Organization. Global recommendations on physical activity for health. Genève: WHO; 2010.

3. World Health Organization. Global status report on noncommunicable diseases 2010. Genève: WHO; 2011.

4. Strong WB, Malina RM, Blimkie CJ, et al. Evidence based physical activity for school-age youth. J Pediatr. 2005;146(6):732-7.

5. Brodersen NH, Steptoe A, Boniface DR, Wardle J. Trends in physical activity and sedentary behaviour in adolescence: ethnic and socioeconomic differences. Br J Sports Med. 2007;41(3):140-4. 
6. Stalsberg R, Pedersen AV. Effects of socioeconomic status on the physical activity in adolescents: a systematic review of the evidence. Scand J Med Sci Sports. 2010;20(3):368-83.

7. Leu $\mathrm{CH}$, Chen KM, Chen HH. A multidimensional approach to child poverty in Taiwan. Child Youth Serv Rev. 2016;66:35-44.

8. Hume C, Salmon J, Ball K. Children's perceptions of their home and neighborhood environments, and their association with objectively measured physical activity: a qualitative and quantitative study. Health Educ Res. 2005;20(1):1-13.

9. Rafaelli M, Koller SH, Cerqueria-Santos E, Morais NA. Developmental risks and psychosocial adjustment among low-income Brazilian youth. Dev Psychopathol. 2007;19(2):565-84.

10. Ré AHN, Logan SW, Cattuzzo MT, Henrique RS, Tudela MC, Stodden DF. Comparison of motor competence levels on two assessments across childhood. J Sports Sci. 2018;36(1):1-6.

11. Bobbio TG, Gabbard C, Gonçalves VG, Filho AA, Morcillo AM. Interlimb coordination differentiates Brazilian children from two socioeconomic settings. Pediatr Int. 2010;52(3):353-7.

12. Valentini NC, Clark JE, Whitall J. Developmental co-ordination disorder in socially disadvantaged Brazilian children. Child Care Health Dev. 2015;41(6):970-9.

13. Hesketh KR, O’Malley C, Paes VM, et al. Determinants of change in physical activity in children 0-6 years of age: a systematic review of quantitative literature. Sports Med. 2017;47(7):1349-74.

14. Lai SK, Costigan SA, Morgan PJ, et al. Do school-based interventions focusing on physical activity, fitness, or fundamental movement skill competency produce a sustained impact in these outcomes in children and adolescents? A systematic review of follow-up studies. Sports Med. 2014;44(1):67-79.

15. Stevens M, Rees T, Coffee P, Steffens NK, Haslam SA, Polman R. A social identity approach to understanding and promoting physical activity. Sports Med. 2017;47(10):1911-8.

16. Alves HPF. Vulnerabilidade socioambiental na metrópole paulistana: uma análise sociodemográfica das situações de sobreposição espacial de problemas e riscos sociais e ambientais. Rev Bras Estud Popul. 2006;23(1):43-59.

17. Serra AS. Pobreza multidimensional no Brasil rural e urbano [tese]. Campinas (SP): Universidade Estadual de Campinas, Instituto de Economia; 2017.

18. Roncancio DJ, Nardocci AC. Social vulnerability to natural hazards in São Paulo, Brazil. Nat Hazards. 2016;84(2):1367-83.

19. Drenowatz C, Eisenmann JC, Pfeiffer KA, et al. Influence of socio-economic status on habitual physical activity and sedentary behavior in 8- to 11-year old children. BMC Public Health. 2010;10:214.

20. Knuth AG, Silva ICM, van Hees VT, et al. Objectively-measured physical activity in children is influenced by social indicators rather than biological lifecourse factors: evidence from a Brazilian cohort. Prev Med. 2017;97:40-4.

21. Cooper AR, Goodman A, Page AS, et al. Objectively measured physical activity and sedentary time in youth: the International children's accelerometry database (ICAD). Int J Behav Nutr Phys Act. 2015;12:113.

22. Penpraze VR, MacLean CM, Montgomery C, et al. Monitoring of physical activity in young children: how much is enough? Pediatr Exerc Sci. 2006;18(4):483-91.

23. van Sluijs EM, Skidmore PM, Mwanza K, et al. Physical activity and dietary behaviour in a population-based sample of British 10-year old children: the SPEEDY study (Sport, Physical activity and Eating behaviour: Environmental Determinants in Young people). BMC Public Health. 2008;8:388.

24. Evenson KR, Catellier DJ, Gill K., Ondrak KS, McMurray R. Calibration of two objective measures of physical activity for children. J Sports Sci. 2008;26(14):1557-65.

25. Centro de Estudos da Metrópole. Mapa da vulnerabilidade social da população da cidade de São Paulo. São Paulo: CEM; 2004.

26. Prefeitura de São Paulo. Favelas situadas no distrito de Ermelino Matarazzo [Internet]. São Paulo: Secretaria Municipal de Habitação; 2015 [acesso 14 nov 2015]. Disponível em: www.habisp.inf.br

27. Empresa Brasileira de Correios e Telégrafos. Estrutura do CEP [Internet]. Brasília: Empresa Brasileira de Correios e Telégrafos; 2016 [acesso em 1 fev 2016]. Disponível em: https://www.correios.com.br/precisa-de-ajuda/o-que-e-cep-e-por-que-usa-lo/ estrutura-do-cep

28. Lohman TG, Roche AF, Martorell R. Anthropometric standardization reference manual. Champaign: Human Kinetics; 1988.

29. Hair Junior JF, Anderson RE, Babin BJ, Black WC. Multivariate data analysis. New Jersey: Prentice Hall; 1998.

30. Verloigne M, Van Lippevelde W, Maes L, et al. Levels of physical activity and sedentary time among 10- to 12-year-old boys and girls across 5 European countries using accelerometers: an observational study within the ENERGY-project. Int J Behav Nutr Phys Act. 2012;9:34.

31. Troiano RP, Berrigan D, Dodd KW, Mâsse LC, Tilert T, McDowell M. Physical activity in the United States measured by accelerometer. Med Sci Sports Exerc. 2008;40(1):181-8. 
32. Baptista F, Santos DA, Silva AM, et al. Prevalence of the Portuguese population attaining sufficient physical activity. Med Sci Sports Exerc. 2012;44(3):466-73.

33. Fairclough SJ, Ridgers ND. Relationships between maturity status, physical activity, and physical self-perceptions in primary school children. J Sports Sci. 2010;28(1):1-9.

34. Aznar S, Naylor PJ, Silva P, et al. Patterns of physical activity in Spanish children: a descriptive pilot study. Child Care Health Dev. 2011;37(3):322-28.

35. Reichert FF, Hallal PC, Wells JC, Horta BL, Ekelund U, Menezes AM. Objectively measured physical activity in the 1993 Pelotas (Brazil) birth cohort. Med Sci Sports Exerc. 2012;44(12):2369-75.

36. Bielemann RM, Cascaes AM, Reichert FF, Domingues MR, Gigante DP. Objectively measured physical activity in children from a southern Brazilian city: a population-based study. J Phys Act Health. 2013;10(8):1145-52.

37. Guinhouya BC, Samouda H, de Beaufort C. Level of physical activity among children and adolescents in Europe: a review of physical activity assessed objectively by accelerometry. Public Health. 2013;127(4):301-11.

38. Fairclough SJ, Ridgers ND, Welk G. Correlates of children's moderate and vigorous physical activity during weekdays and weekends. J Phys Act Health. 2012;9(1):129-37.

39. Brooke HL, Corder K, Atkin AJ, van Sluijs EM. A systematic literature review with meta-analyses of within- and between-day differences in objectively measured physical activity in school-aged children. Sports Med. 2014;44(10):1427-38.

40. Kettner S, Kobel S, Fischbach N, et al. Objectively determined physical activity levels of primary school children in south-west Germany. BMC Public Health. 2013;13:895.

41. Guinhouya CB, Hubert H, Soubrier S, Vilhelm C, Lemdani M, Durocher A. Moderate-to-vigorous physical activity among children: discrepancies in accelerometry-based cut-off points. Obesity. 2006;14(5):774-7.

42. Kim Y, Beets MW, Welk GJ. Everything you wanted to know about selecting the "right" Actigraph accelerometer cut-points for youth, but...: a systematic review. J Sci Med Sport. 2012;15(4):311-21.

43. Nader PR, Bradley RH, Houts RM, McRitchie SL, O’Brien M. Moderate-to-vigorous physical activity from ages 9 to 15 years. JAMA. 2008;300(3):295-305.

44. Riddoch CJ, Bo Andersen L, Wedderkopp N, et al. Physical activity levels and patterns of 9-and 15-yr-old European children. Med Sci Sports Exerc. 2004;36(1):86-92.

45. Cohen KE, Morgan PJ, Plotnikoff RC, Callister R, Lubans DR. Fundamental movement skills and physical activity among children living in low-income communities: a cross-sectional study. Int J Behav Nutr Phy. 2014;11(1):49.

46. Carson RL, Castelli DM, Kuhn ACP, et al. Impact of trained champions of comprehensive school physical activity programs on school physical activity offerings, youth physical activity and sedentary behaviors. Prev Med. 2014;69(1):S12-9.

47. Laukkanen A, Pesola A, Havu M, Sääkslahti A, Finni T. Relationship between habitual physical activity and gross motor skills is multifaceted in 5- to 8-year-old children. Scandinavian J Med Sci Sports. 2014;24(2):102-10.

48. Trost SG, Loprinzi PD, Moore R, Pfeiffer KA. Comparison of accelerometer cut points for predicting activity intensity in youth. Med Sci Sports Exerc. 2011;43(7):1360-8.

49. Bornstein DB, Beets MW, Byun W, et al. Equating accelerometer estimates of moderate-to-vigorous physical activity: in search of the Rosetta Stone. J Sci Med Sport. 2011;14(5):404-10.

50. Beenackers MA, Kamphuis CB, Giskes K, et al. Socioeconomic inequalities in occupational, leisure-time, and transport related physical activity among European adults: a systematic review. Int J Behav Nutr Phys Act. 2012;9:116.

51. Bosdriesz JR, Witvliet MI, Visscher TL, Kunst AE. The influence of the macro-environment on physical activity: a multilevel analysis of 38 countries worldwide. Int J Behav Nutr Phys Act. 2012;9:110.

52. Gonçalves H, Hallal PC, Amorim TC, Araújo CLP, Menezes AMB. Fatores socioculturais e nível de atividade física no início da adolescência. Rev Panam Salud Publica. 2007;22(4):246-53.

53. Farias Júnior JC. Prevalência e fatores de influência para inatividade física em adolescentes. Rev Bras Cien Mov. 2006;14(1):63-70.

54. Ridgers ND, Stratton G, Fairclough SJ. Physical activity levels of children during school playtime. Sports Med. 2006;36(4):359-71.

55. Blum WB, McNeeley C, Nonnemaker J. Vulnerability, risk, protection. J Adolesc Health. 2002;31(1):28-39.

56. Copetti J, Neutzling MB, Silva MC. Barreiras à prática de atividades físicas em adolescentes de uma cidade do sul do Brasil. Rev Bras Ativ Fis Saúde. 2012;15(2):88-94.

57. Abbasi IN. Socio-cultural barriers to attaining recommended levels of physical activity among females: a review of literature. Quest. 2014;66(4):448-67.

58. Colabianchi N, Griffin JL, McIver KL, Dowda M, Pate RR. Where are children active and does it matter for physical activity? A latent transition analysis. J Phys Act Health. 2016;13(12):1294-300. 
59. Kelly LA, Reilly JJ, Fisher A, et al. Effect of socioeconomic status on objectively measured physical activity. Arch Dis Child. 2006;91(1):35-8.

60. Tandon PS, Zhou C, Sallis JF, Cain KL, Frank LD, Saelens BE. Home environment relationships with children's physical activity, sedentary time, and screen time by socioeconomic status. Int J Behav Nutr Phys Act. 2012;9:88.

61. Vandendriessche JB, Vandorpe BF, Vaeyens R, et al. Variation in sport participation, fitness and motor coordination with socioeconomic status among Flemish children. Pediatr Exerc Sci. 2012;24(1):113-28.

62. Choi H, Burgard S, Elo IT, Heisler M. Are older adults living in more equal counties healthier than older adults living in more unequal counties? A propensity score matching approach. Soc Sci Med. 2015;141:82-90.

63. Doom JR, Mason SM, Suglia SF, Clark CJ. Pathways between childhood/adolescent adversity, adolescent socioeconomic status, and long-term cardiovascular disease risk in young adulthood. Soc Sci Med. 2017;188:166-75.

64. Butte NF, Puyau MR, Adolph AL, Vohra FA, Zakeri I. Physical activity in nonoverweight and overweight Hispanic children and adolescents. Med Sci Sports Exerc. 2007;39(8):1257-66.

65. Lampard AM, Jurkowski JM, Lawson HA, Davison KK. Family ecological predictors of physical activity parenting in low income families. Behav Med. 2013;39(4):97-103.

66. Ferreira I, van der Horst K, Wendel-Vos W, Kremers S, van Lenthe FJ, Brug J. Environmental correlates of physical activity in youth: a review and update. Obes Rev. 2007;8(2):129-54.

67. Gordon-Larsen P, Nelson MC, Page P, Popkin B. Inequality in the built environment underlies key health disparities in physical activity and obesity. Pediatrics. 2006;117(2):417-24.

68. Telama R, Yang X, Hirvensalo M, Raitakari O. Participation in organized youth sport as a predictor of adult physical activity: a 21-year longitudinal study. Pediatr Exerc Sci. 2006;17(1):76-88.

69. Vander Ploeg KA, Maximova K, McGavock J, Davis W, Veugelers P. Do school-based physical activity interventions increase or reduce inequalities in health? Soc Sci Med. 2014;112:80-7.

ENDEREÇO:

Alessandro H. Nicolai Ré

Rua Arlindo Bettio, 1000 - 03828000

São Paulo - BRASIL e-mail: alehnre@usp.br
Recebido para publicação: 19/11/2015

$1^{a}$ Revisão: 01/10/2016

$2^{\text {a }}$ Revisão: 29/09/2017

Aceito: 20/12/2017 